\title{
Cough and fever in a female with Crohn's disease receiving infliximab
}

\section{G. Stratakos*, I. Kalomenidis*, V. Papas*, K. Malagari", A. Kollintza*, C. Roussos*, M. Anagnostopoulou\#, O. Paniara*, S. Zakynthinos* and S.A. Papiris ${ }^{+}$}

\section{CASE HISTORY}

A 77-yr-old housewife, who was a never-smoker, presented at the Evangelismos General Hospital (Athens, Greece), complaining of anorexia, cough, fever of $39^{\circ} \mathrm{C}$, dyspnoea, severe weakness and dysuria for the past 10 days.

She had reported poor health status during the preceeding month. The patient had been diagnosed with Crohn's disease 9 months earlier. Initially, the patient was treated with $32 \mathrm{mg} \cdot \mathrm{d}^{-1}$ methylprednisolone and $100 \mathrm{mg} \cdot \mathrm{d}^{-1}$ azathioprine, both taken orally. Azathioprine was discontinued 4 weeks later, due to hepatic damage, and was replaced by infliximab injections; the chimeric monoclonal immunoglobulin (Ig)-G1 antibody to tumour necrosis factor (anti-TNF)- $\alpha$. Treatment was initially $5 \mathrm{mg} \cdot \mathrm{kg}^{-1}$ at 0,2 and 6 weeks as an induction regimen, followed by a maintenance regimen of $5 \mathrm{mg} \cdot \mathrm{kg}^{-1}$ every 8 weeks, the latter one taken 10 days before admission. In total, the patient received six doses of anti-TNF- $\alpha$ over a period of 8 months. At the same time, she also received $16 \mathrm{mg} \cdot \mathrm{d}^{-1}$ of methylprednisolone orally.

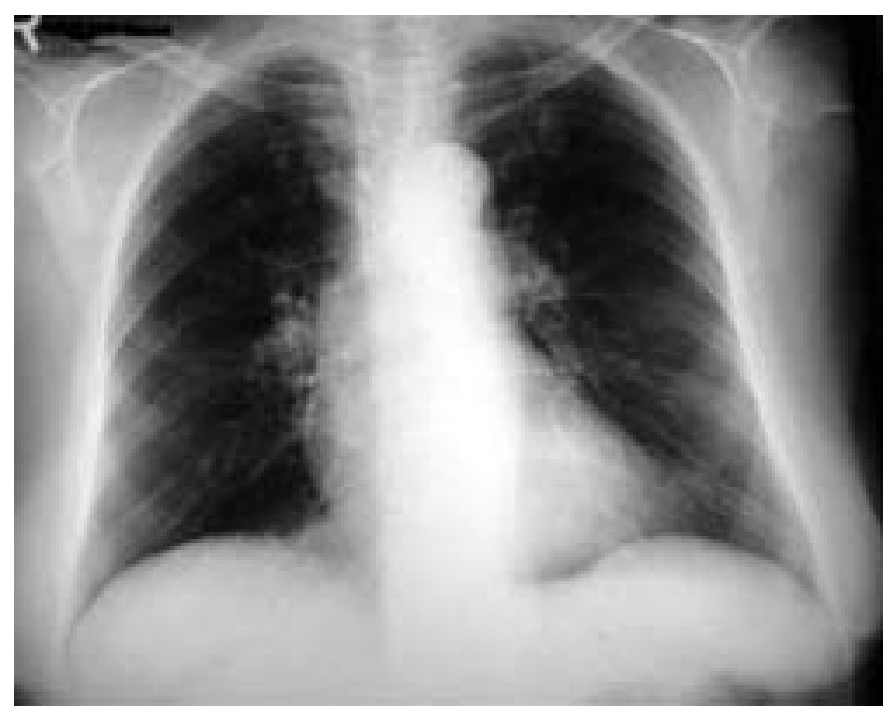

FIGURE 1. Posteroanterior radiograph of the chest, obtained 3 weeks before admission.
The patient also suffers from type II diabetes mellitus for which she was receiving glybenclamide orally, as well as arterial hypertension, which was controlled with an oral acetylcholine esterase inhibitor. The patient's past medical history records showed acute hepatitis 40 yrs previously, hysterectomy with ovariectomy 25 yrs previously and cholecystectomy 5 yrs previously.

On physical examination, the patient was severely ill, with a body temperature of $38.5^{\circ} \mathrm{C}$, pulse rate of 110 beats $\cdot \mathrm{min}^{-1}$, blood pressure $130 / 80 \mathrm{mmHg}$ and respiratory rate of 28 breaths $\cdot \mathrm{min}^{-1}$. On chest auscultation, rales were audible from both hemithoraces and there were no heart murmurs. Oropharyngeal thrush was clearly evident. The abdomen was distended and slightly sensitive on palpation; however, intestinal sounds were normal. She had no clubbing and the rest of the physical examination was unremarkable.

Laboratory investigations revealed the following: haemoglobin $118 \mathrm{~g} \cdot \mathrm{L}^{-1}$; total leukocyte count $12.6 \times 10^{9} \cdot \mathrm{L}^{-1}(93 \%$ neutrophils); haematocrit 0.34 ; and a platelet count of

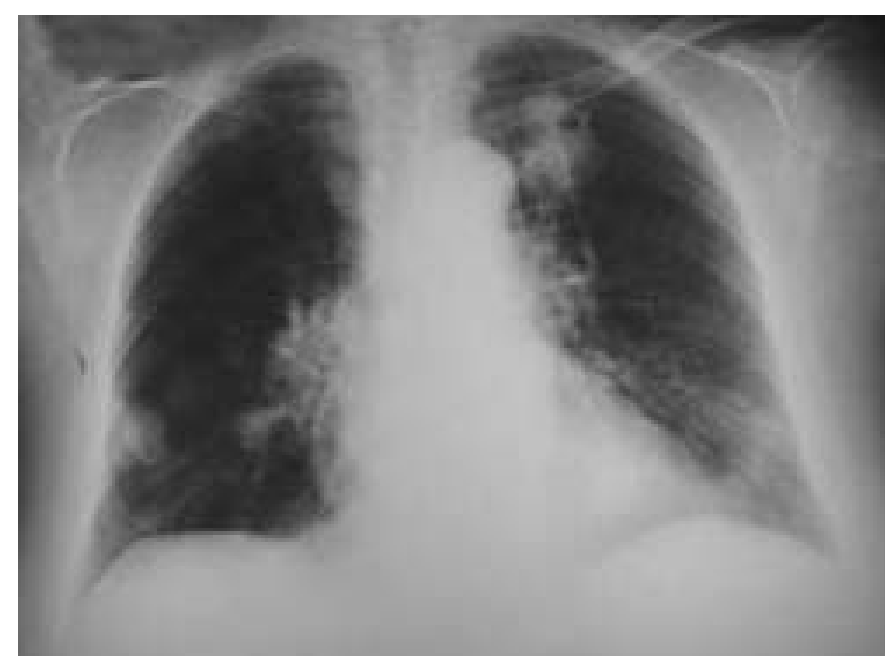

FIGURE 2. Posteroanterior radiograph of the chest, obtained on the day of admission.

\footnotetext{
*Dept of Critical Care and Pulmonary Services, and "Dept of Microbiology, Evangelismos Hospital, and \#2nd Dept of Radiology, and +2nd Pulmonary Dept, National and Capodistrian University of Athens, Attikon University Hospital, Athens, Greece.

CORRESPONDENCE: S.A. Papiris, 2nd Dept of Pulmonary Medicine, 1 Rimini Street, 12462 Haidari, Attikon University Hospital, Athens, Greece. Fax: 30 2105326414. E-mail: papiris@otenet.gr
} 

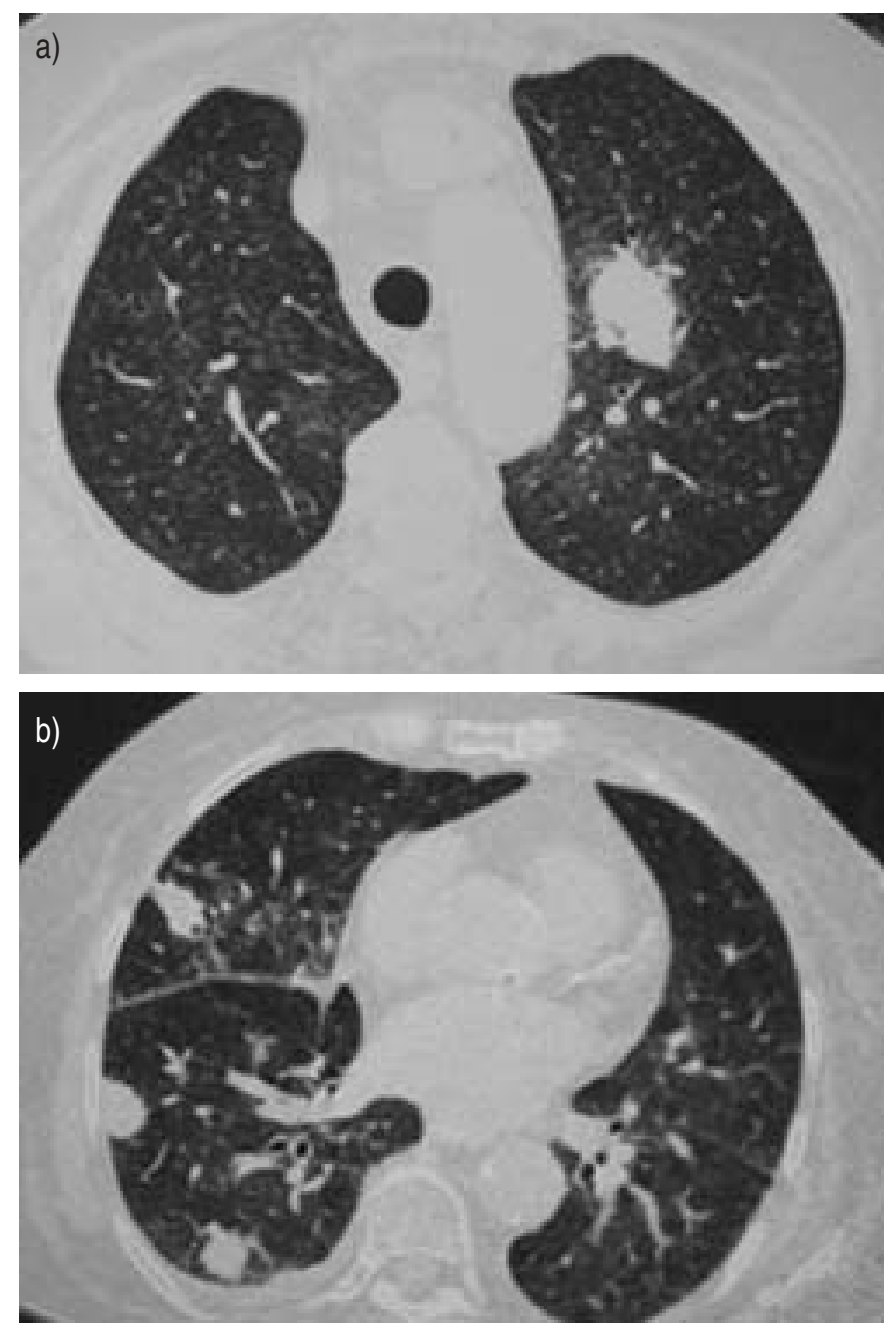

FIGURE 3. Thin computed tomography section at the level of the a) upper lobes on the day of admission and b) right inferior pulmonary vein on the day of admission

$370 \times 10^{9} \cdot \mathrm{L}^{-1}$. The alanine aminotransferase level was $21.8 \mu \mathrm{kat} \cdot \mathrm{L}^{-1}$ and $\gamma$-glutamyl-transferase $393 \mathrm{U} \cdot \mathrm{L}^{-1}$. The rest of the serum chemistries were within normal range. Serological tests for viral hepatitis revealed previous hepatitis B infection: hepatitis B surface antigen (HBs Ag) (-), anti-HBs (+), antihepatitis B early antigen (+), and anti-hepatitis B core antigen $(+)$. An ELISA test for anti-HIV antibodies was negative. The oral swab, cultures of blood, sputum and urine, urine Legionnella antigen, as well as the serological tests for Mycoplasma spp., Chlamydia spp., Ricketsiae spp., malaria, Leishmania spp., Brucellosis spp. and common virus were immediately requested and were pending. A tuberculin test was performed, with $2 \mathrm{iu}$ PPD RT 23 tuberculin, on the day of admission; results were negative $(0 \mathrm{~mm})$. Arterial blood gases

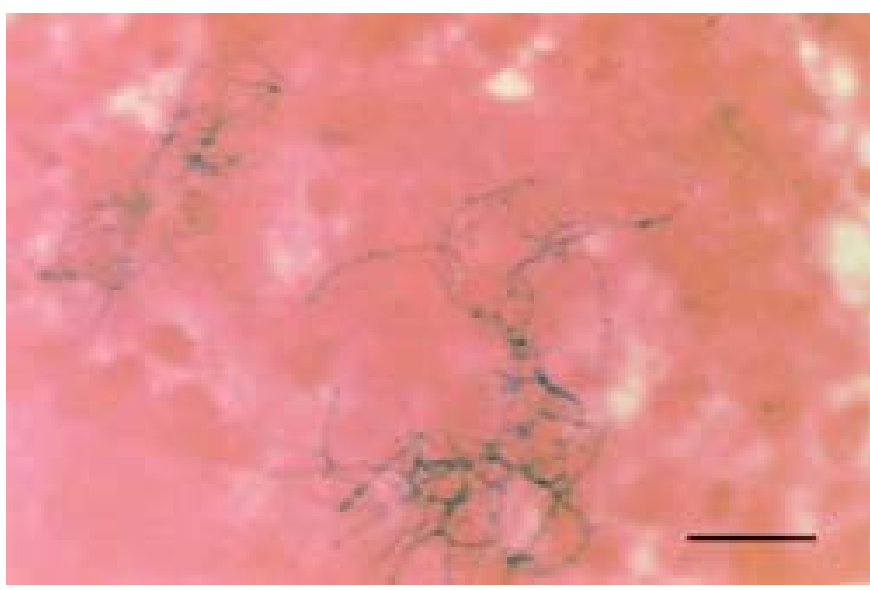

FIGURE 4. Bronchoalveolar lavage fluid specimen, using the modified Kinyoun method stain. Scale bar $=1 \mu \mathrm{m}$.

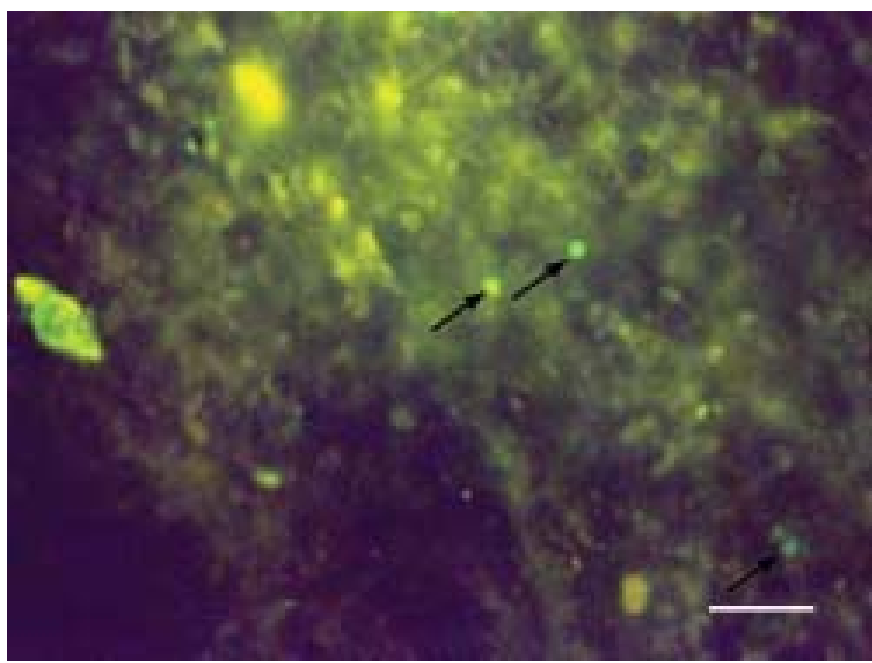

FIGURE 5. Bronchoalveolar lavage fluid specimen using indirect qualitative immunofluorescence. Arrows indicate a positive result. Scale bar $=2 \mu \mathrm{m}$.

on room air were as follows: arterial oxygen tension $7.33 \mathrm{kPa}$; carbon dioxide arterial tension $4.39 \mathrm{kPa}$; and $\mathrm{pH} 7.44$.

A posteroanterior radiograph of the patient's chest was carried out in another hospital 3 weeks before her admission and is shown in figure 1 . The posteroanterior chest radiograph obtained on admission is also shown in figure 2. The thinsection computed tomography (CT) scan of the thorax on the same day is shown in figure 3.

A fibreoptic bronchoscopy and bronchoalveolar lavage (BAL) of the right middle lobe was performed on the day after admission. The stains on BAL samples revealed diagnostic findings, which are shown in figures 4 and 5 .

\section{BEFORE TURNING THE PAGE, INTERPRET THE PATIENT'S HISTORY, CHEST RADIOGRAPHS, COMPUTED TOMOGRAPHY SCAN AND MICROBIOLOGICAL STAINS, AND SUGGEST A DIAGNOSIS.}




\section{INTERPRETATION}

\section{Chest radiographs and CT scans}

The posteroanterior radiograph of the chest in figure 1, obtained 3 weeks before admission, showed a small left upper lobe nodule. The posteroanterior radiograph of the chest in figure 2, obtained on the day of admission, showed multiple nodules in both lungs. The thin section computed tomography in figure 3 , obtained on the day of admission, showed an illdefined opacity on the left as the level of the aortic arch measuring approximately $2 \times 3 \mathrm{~cm}$ with an air bronchogram, scattered small nodules bilaterally and multiple nodular opacities at the level of the right inferior pulmonary vein. Patchy ground-glass opacities were seen, as well as a small pleural effusion in the right.

\section{Microbiology}

In figure 4, BAL fluid specimen showed partially acid-fast crooked, branching, beaded, filaments stained by the modified Kinyoun method, typical for Nocardia spp. In figure 5, BAL fluid specimen, using indirect qualitative immunofluorescence test using murine anti-Pneumocystis jiroveci monoclonal antibody, indicated the typical microscopic morphology of Pneumocystis jiroveci, with characteristic clear-cut round shaped apple-green fluorescence.

Diagnosis: Opportunistic lung co-infection caused by Nocardia asteroides and Pneumocystis jiroveci.

\section{TREATMENT AND CLINICAL COURSE}

After the microbiological diagnosis of pulmonary nocardiosis and Pneumocystis jiroveci (formerly Pneumocystis carinii) pneumonia was made. The patient was treated with $15 \mathrm{mg} \cdot \mathrm{kg}^{-1} \cdot \mathrm{day}^{-1}$ of trimethoprim and $75 \mathrm{mg} \cdot \mathrm{kg}^{-1} \cdot \mathrm{day}^{-1}$ of sulphamethoxazole (co-trimoxazole) intravenously. This is the treatment of choice against both pathogens.

The oral swab disclosed an associated Candida albicans oropharyngeal infection, and the sputum and urine cultures disclosed Acinetobacter baumanii- and Proteus mirabilis- associated airways and urinary infections, respectively. Cotrimoxazole treatment was coupled with oral administration of voriconazole $200 \mathrm{mg}$ b.i.d for the treatment of Candida albicans oropharyngeal mycosis. Proteus mirabilis urinary tract and the Acinetobacter baumanii airways infections were both treated with meropenem $1 \mathrm{~g}$ t.i.d by i.v. infusion according to the results of the microbiological sensitivity tests.

Oesophagogastroduodenal endoscopic examination, in addition to the colonoscopic examination, did not reveal any abnormal changes. The patient showed gradual clinical improvement. The lung nodular lesions on the chest radiographs, initially cavitated and subsequently resolved. Cotrimoxazole was administered orally after an initial i.v. treatment of 6 weeks. Voriconazole and meropenem were discontinued after 2 weeks. The patient was dismissed from the hospital 2 months after admission and continued to receive a reduced dosage of co-trimoxazole until the completion of a total treatment time of 6 months.

\section{DISCUSSION}

Infliximab is an intravenously administered chimeric monoclonal Ig-G1 antibody to TNF- $\alpha$. It is composed of human-constant and murine-variable regions. TNF- $\alpha$, a proinflammatory cytokine, is mainly produced by activated macrophages and T-lymphocytes, and is a key cytokine in Crohn's disease and other chronic inflammatory conditions, including rheumatoid arthritis and psoriasis [1]. TNF- $\alpha$ induces other pro-inflammatory cytokines, including interleukin (IL)-1 and IL-6, and enhances leukocyte migration by inducing expression of adhesion molecules in both endothelial cells and leukocytes. TNF- $\alpha$ also activates leukocytes and induces acute-phase reactants and metalloproteinases.

Infliximab was first used in Crohn's disease in 1993 by DERKX et al. [2] and proved lifesaving in a desperate case, changing the treatment of the disease. It is indicated in refractory, luminal and fistulising Crohn's disease, and several thousands of patients are treated worldwide. The mechanism of action of infliximab is not well understood and probably extends far beyond the mere neutralisation of TNF- $\alpha$. It can block other pro-inflammatory signals or molecules, decreasing the number of neutrophils. Additionally, it can induce apoptosis of various inflammatory cells carrying membrane-bound TNF- $\alpha$ [1]. However, given the complexity of cytokine interactions and the multiplicity of cytokine targets, infliximab-associated immunomodulation is very likely to lead to a higher risk of severe infections. Disseminated infections may arise, especially reactivation of latent tuberculosis and herpes viruses, as well as development of several fungal infections, including Pneumocystis jiroveci pneumonia and severe sepsis from both Gram-positive and Gram-negative microorganisms [3-8]. This risk is even greater if the patients are concomitantly treated with corticosteroids or other immunosuppressants [1]. However, because of physicians under reporting, it is estimated that $<5 \%$ of infliximab-associated infections are documented. The true incidence of infections caused by infliximab-related immunosuppression or other similar treatments, both serious and nonserious, remains unknown [3].

The patient reported here was heavily immunosuppressed, receiving both infliximab and glucocorticosteroids. She developed multiple, concomitant and severe opportunistic infections of the upper and lower respiratory tract, coupled with an infection of the urinary system. To the best of the current authors' knowledge, this is the first case of dual pulmonary infection by Nocardia asteroides and Pneumocystis jiroveci associated with infliximab treatment for inflammatory bowel disease.

Nocardia asteroides pneumonia is by far the most common respiratory tract nocardial disease, and, typically, it has a subacute course. Single or multiple nodules are common radiographical presentations, and present a high tendency to cavitate. The patient presented with an abnormal chest radiograph 3 weeks before admission, which was characterised by a single nodule in the left upper lobe that was overlooked by her physicians. The paucity of respiratory symptoms might have contributed to misdiagnosis, although this radiographical picture is characteristic for Nocardia asteroides infection and also for other infections developing in patients receiving anti-TNF- $\alpha$ treatment [8]. Subsequently, the patient developed multiple and bilateral lung nodules that rapidly cavitated after the institution of effective treatment, and gradually disappeared. 
Pneumocystis jiroveci, another pathogen that affected the patient, is an important, but sporadic, opportunistic pathogen in immunosuppressed HIV seronegative individuals [9]. Patients with Pneumocystis jiroveci pneumonia develop dyspnoea, fever and nonproductive cough. Symptoms may be relatively subtle, especially in severely immunocompromised patients, and may last for a few weeks. In the present patient, Pneumocystis jiroveci pneumonia was detected early, since radiographical signs compatible with this infection included only a moderate and sparse "ground-glass" appearance of both lungs. Experimental studies have shown that TNF- $\alpha$ is important in the host defences against both pathogens, Nocardia asteroides and Pneumocystis jiroveci [10, 11].

Candida albicans is a normal inhabitant of the mouth and can be recovered from sputum of $20-55 \%$ of normal subjects. Candida spp. oropharyngeal infection was not surprising, since infliximab tends to complicate fungal infections. Voriconazole was chosen as fungal pneumonia could not be initially excluded, and an invasive surgical approach to obtain lung tissue (the gold standard for its diagnosis) could not be performed because of the patient's critical clinical condition. However, prompt and plausible diagnoses were obtained by fibreoptic bronchoscopy and BAL, a day after admission [12].

Acinetobacter baumanii airways infection, another potentially serious infection in the immunocompromised, was diagnosed by quantitative sputum cultures, and was coupled with another Gram-negative infection, that of Proteus mirabilis urinary tract infection. Both infections were treated adequately with the same antibiotic. Fibreoptic bronchoscopy and BAL were again of paramount importance for the early establishment of the diagnosis and permitted prompt and effective treatment.

In conclusion, patients receiving anti-tumour necrosis factor- $\alpha$ therapy are at risk for developing multiple, at times concomitant, and severe opportunistic infections from various microbial, viral and fungal pathogens, including Nocardia asteroides and Pneumocystis jiroveci. The severity and the multiplicity of opportunistic infections observed in the patient presented here and in others receiving infliximab suggest that additional strategies need to be established for the prevention, rapid identification and preventive treatment of such infections.

\section{REFERENCES}

1 Rutgeerts P, Assche G, Vermeire S. Optimizing anti-TNF treatment in inflammatory bowel disease. Gastroenterology 2004; 126: 1593-1610.

2 Derkx B, Taminiau J, Radema S, et al. Tumor-necrosisfactor antibody treatment in Crohn's disease. Lancet 1993; 342: 173-174.

3 Dinarello CA. Anti-cytokine therapeutics and infections. Vaccine 2003; 21: Suppl. 2, s24-s34.

4 Giles JT, Bathon JM. Serious infections associated with anticytokine therapies in the rheumatic diseases. J Intensive Care Med 2004; 19: 320-334.

5 Keane J, Gershon S, Wise RP, et al. Tuberculosis associated with infliximab, a tumor necrosis factor $\alpha$-neutralizing agent. N Engl J Med 2001; 345: 1098-1104.

6 van der Klooster JM, Bosman RJ, Oudemans van Straaten HM, van Spoel Ji, Wester JP, Zandstra DF. Disseminated tuberculosis, pulmonary aspergillosis and cutaneous herpes simplex infection in a patient with infliximab and methotrexate. Intensive Care Med 2003; 29: 2327-2329.

7 Wood KL, Hage CA, Knox KS, et al. Histoplasmosis after treatment with anti-tumor necrosis factor- $\alpha$ therapy. Am J Respir Crit Care Med 2003; 167: 1279-1282.

8 Kroesen S, Widmer AF, Tyndal A, Hasler P. Serious bacterial infections in patients with rheumatoid arthritis under ant-TNF- $\alpha$ therapy. Rheumatology 2003; 42: 617-621.

9 Russian DA, Levine SJ. Pneumocystis carinii pneumonia in patients without HIV infection. Am J Med Sci 2001; 321: 56-65.

10 Silva CL, Faccioli LH. Tumor necrosis factor and macrophage activation are important in clearance of Nocardia brasiliensis from the livers and spleens of mice. Infect Immunol 1992; 60: 3566-3570.

11 Chen W, Havell EA, Harmsen AG. Importance of endogenous tumor necrosis factor- $\alpha$ and $\gamma$ interferon in host resistance against Pneumocystis carinii infection. Infect Immun 1992; 60: 1279-1284.

12 Pappas PG, Rex JH, Sobel JD, Filler SG, Dismukes WE, Waish TJ. Guidelines for treatment of Candidiasis. CID 2004; 38: 161-189. 